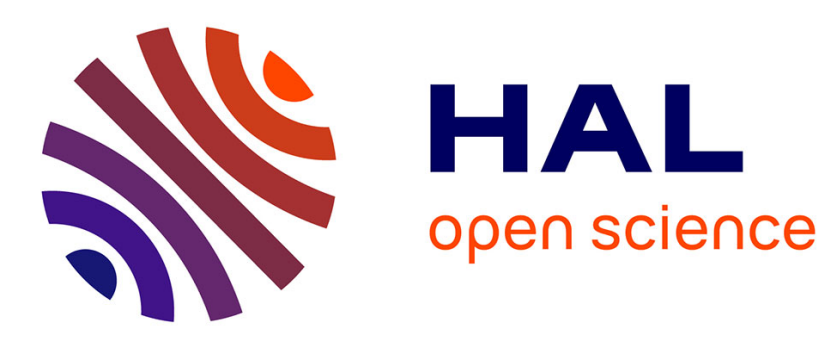

\title{
Design Patterns for Modelling Life Trajectories in the Semantic Web
}

David Noel, Marlène Villanova-Oliver, Jérôme Gensel, Pierre Le Quéau

\section{To cite this version:}

David Noel, Marlène Villanova-Oliver, Jérôme Gensel, Pierre Le Quéau. Design Patterns for Modelling Life Trajectories in the Semantic Web. International Symposium on Web and Wireless Geographical Information Systems (W2GIS 2017), May 2017, Shangai, China. pp.51-65, 10.1007/978-3-319-559988_4. hal-01585043

\section{HAL Id: hal-01585043 https://hal.science/hal-01585043}

Submitted on 11 Sep 2017

HAL is a multi-disciplinary open access archive for the deposit and dissemination of scientific research documents, whether they are published or not. The documents may come from teaching and research institutions in France or abroad, or from public or private research centers.
L'archive ouverte pluridisciplinaire HAL, est destinée au dépôt et à la diffusion de documents scientifiques de niveau recherche, publiés ou non, émanant des établissements d'enseignement et de recherche français ou étrangers, des laboratoires publics ou privés. 


\title{
Design patterns for modelling life trajectories in the semantic web
}

\author{
David Noël ${ }^{1}$, Marlène Villanova-Oliver ${ }^{1}$, Jérôme Gensel ${ }^{1}$, Pierre Le Quéau ${ }^{2}$ \\ 1. Univ. Grenoble Alpes, LIG \\ F-38000 Grenoble, France \\ name.firstnamedimag.fr \\ 2. Univ. Grenoble Alpes, PACTE \\ F-38000 Grenoble, France \\ pierre.le-queauduniv-grenoble-alpes.fr
}

\begin{abstract}
.
Most of the existing approaches for trajectory modelling propose to enrich structured spatiotemporal trajectories with semantics. In social sciences, the term of "trajectory" is often used to describe some evolution that is not necessarily related to some geographical movement. In this paper, we propose ontological design patterns that i) allow modelling multiple spatial or aspatial trajectories and ii) include explanatory factors for a better understanding of trajectory events. Algorithms for the exploitation of our patterns are also presented. As a case study, we model the multiple trajectories that compose a life trajectory having in mind to focus on and study residential choices. This is an important issue for decision makers and urban planning experts in metropolitan areas who need to better understand choices citizens make. We show how our trajectory model, once instantiated, can be exploited using temporal, spatial and thematic dimensions.
\end{abstract}

Keywords: Trajectory modelling, life trajectory, metaphorical trajectories, design patterns, semantic web, residential choices.

\section{Introduction}

Urban planning experts and decision makers face many challenges related to residential choices made by inhabitants of metropolitan areas. These choices are strongly influenced by the various phases individuals go through in their personal lives. As an example, in general and in Europe, couples with children prefer to live far from the metropolitan centers [5]. This exodus of working couples with (young) children towards peri-urban areas is one of the causes of important problems major cities have to solve in the field of urban planning. For instance, it leads satellite cities in the periphery of such metropolitan areas to provide different kinds of amenities (for instance, nurseries and schools) with adapted opening hours, but it also increases the time workers need to commute from home to work, which generates pollution and traffic congestion... 
Reasons for residential choices can be better understood through a comprehensive approach for life trajectories of individuals that takes into account many decisive aspects of citizens' lives: familial, professional, etc. Then, in order to study the residential trajectory of an individual (the succession of its residential choices in time and space), such a trajectory should be considered as an integrated part of a broader vision of her/his life trajectory [19]. The life trajectory of an individual is thus composed of multiple semantic trajectories, each depending on a particular thematic point of view (residential, professional, familial...). The term "trajectory" obviously evokes a spatial dimension. If, most of the time, the spatiality of a trajectory relates to a geographical reference system, it sometimes refers to more abstract spaces, like trajectories studied in sociology and, more widely, in social sciences. For instance the notion of career defined by Howard Becker [4], "refers to the sequence of movements from one position to another". If the analogy with a geographical space is strong, we nevertheless consider that some trajectories are "ageographical", meaning that they cannot be exactly considered as geographical ones. A second point is that such a professional trajectory includes "both objective facts of social structure and changes in the viewpoints, motivations, and desires of the individual". Thus for Grafmeyer and Authier [6], a residential trajectory "tends to suggest that successive positions in a given set is not simply by chance, but rather are linked together by an intelligible order". Part of our objective is to elicit the explanatory factors or combinations of factors that have shaped a given life trajectory.

In the case of residential trajectories, we want to bring to light the underlying reasons why individuals move to a given place, at a specific moment of their lives. External factors can be found in the context surrounding the life trajectory (the evolution of the housing market or some changes in the surroundings of the housing, etc.). Internal factors are related to life circumstances of the individual: they are directly linked to the specific characteristics of one or more points of view in her/his life trajectory. For instance, one may observe that a family moves for a new apartment with an additional room because of a birth to come. Here, a residential event (the move) is - partly explained by an event belonging to the family point of view. In this context, our work focuses specifically on highlighting cause and effect relationships between events that arise in the life of individuals.

One of our objectives is first to integrate in the same representation the multiple thematic points of view that belong to a single life trajectory, whether they are spatial or aspatial. Further, we plan to analyse and to compare parts of trajectories, whether they belong to the same or to different persons (e.g. compare someone's residential and professional trajectories, or compare residential trajectories of two individuals). To easily combine (parts of) trajectories, we need to represent them using the same concepts to structure information and, as far as possible, well-known frames of reference to characterize it. This latter point in particular encompasses time and space representation. We thus propose an approach based on ontology design patterns $\left(\mathrm{ODP}^{1}\right)$ compatible with Semantic Web initiatives such as Timeline Ontology [14] or GeoSPARQL [13]. One of these ODP allows modelling both spatial and aspatial specific trajectories (i.e.

\footnotetext{
${ }^{1}$ http://ontologydesignpatterns.org/
} 
a residential trajectory, a professional trajectory, etc.) as parts of one life trajectory ontology. A second ODP is dedicated to the representation of explanatory factors. Gathering these design patterns together and applying them as many times as needed in a design process leads to the production of an ontological representation of the different points of view of people's life trajectory, including explanation about choices they made. Once filled, such an ontology can be used to explore, analyse and compare life trajectories.

This paper is organized as follows. In section 2, we present related works that laid the foundation of our work. In section 3, we describe the ontological design patterns (in RDF) we propose to build ontological models of trajectories composed of multiple thematic points of view, and that include explanatory factors for a better understanding of trajectory events. In section 4 , we present a set of algorithms that define how to produce a model (i.e. an RDF ontology) from our patterns. We show in section 5 how the obtained ontology can be exploited (i.e. fulfilled and queried), before we conclude.

\section{Related work}

\subsection{Semantic Trajectory modelling}

Many semantic trajectory models have been proposed in the recent years, offering different solutions to enrich space-time trajectories of mobile objects ${ }^{2}$. Most of these models are based on a spatiotemporal segmentation of trajectories. For example, in one of the major works in the field [17], that follow Hägerstraand [7] and the "time-geography", these trajectories are structured through the concept of stop, which characterizes a part of a spatiotemporal trajectory where the object's position stays fixed, and the concept of move where, contrariwise, its position changes in time. Information about the trajectory is then added to these structures, such as the nature of the moving object, the mode of transportation during a move, or the visited places at a stop. This model is particularly adapted for characterizing a semantic evolution that is related to movements in a geographical space. Other researchers have modelled spatio-temporal trajectories through periods of trips and periods of activities [20].

Other design patterns, connected to the Semantic Web, have also been proposed. For example, [8] present a geo-ontology design pattern that allows describing a semantic trajectory as a sequence of spatiotemporal points, and which makes use of widely known upper level ontologies. In this pattern, the concept of segment is used to represent a portion of trajectory that gathers fixes together. A fix here is given as a spatiotemporal point indicating the position of a moving object at one instant in time. A. Krisnadhi et al. [10] propose a generalization ${ }^{3}$ of this pattern by employing the notion of place, instead of location/geo-coordinate, to represent the location associated with a fix (this confirms the position defended in [8] that no granularity is imposed for the location associated with a fix). Compared to [17], at a conceptual level, a segment can

\footnotetext{
${ }^{2}$ Mobile objects can be of different sorts including people

${ }^{3} \mathrm{http}$ ://ontologydesignpatterns.org/wiki/Submissions:Trajectory
} 
cover the meaning of both stop and move concepts (considering that, at the logical level, the spatiotemporal information is not associated with the segment itself but rather with the fixes).

All these works propose a generic, high-level representation of trajectories. They mainly address the description of trajectories that have a strong spatiotemporal dimension, with an anchoring in a geographical space, and in which both the periods of stability and of movement can be represented. It has to be noted that semantics is added using attributes attached to the main concepts of the trajectory (stop, move, segment, fix, etc.).

In [17], the authors also point out that: "the term trajectory is sometimes used in a metaphorical sense to describe an evolution, although the evolution at hand is not related to physical movement". They call this kind of trajectories metaphorical trajectories in reference to "the idea of an object (e.g. the person) moving in an abstract space" and give the example of $a$ career trajectory to illustrate it. Then they propose to simply model such a metaphorical trajectory using the conceptual model based on stop and move, transposing these two notions in the so-called abstract space. In this case, a given position in the abstract space is characterized by a stop in the trajectory. However, although the authors say nothing about it, it seems to us that the concept move has here a limited meaning in such metaphorical trajectories: the movement characterized by a move is difficult to translate in the abstract space (e.g. what does moving from one job position to another means from a spatial and temporal points of view?). In [16], Raubal also propose an approach based on time geography that allow to characterize an evolution of semantic over time in an abstract space. The proposed approach rest on an algebraic model and is not a design pattern using semantic web technology.

The other works we cite in section 2.1 do not concretely address this issue of so-called metaphorical trajectories that are important in our context. In [8], the authors mention that social sciences fields (psychologists, anthropologists, geographers, traffic planners) have investigated human trajectories to better understand human behaviour. Nevertheless, the example of a human trajectory they give, based on their pattern, deals with a daily individual's trajectory data recorded by a handheld GPS receiver (i.e. is not a metaphorical trajectory and not a life-long trajectory).

The issues we address in this paper are closely related to previous approaches on life trajectory modelling, which refine and adapt the notion of trajectory to a specific context. In particular, the work conducted by Thériault et al. is a reference in this field. The space-time model for the analysis of life trajectories [18] is based on three different trajectories - residential, family and professional. Each of these trajectories is conceptually modelled by episodes - corresponding to stable statutes during a time interval and events which alter one or more of these statutes. The conceptual model is based on a relational approach. Later, the model has been modified in order to determine the likelihood of an event occurring under certain conditions in a life trajectory [19]. These models are centred on the temporal aspect of residential choices: the focus is more on when people have moved at some place (depending on some life circumstances) than 
on the reasons why they have chosen the place where they have moved to.

Regarding our objective, these works show to have some limitations. First, in [18, 19], the support to the representation of integrated multiple trajectories is limited to three predefined points of view. If one could imagine that some other dimension could be added, the authors do not provide any methodological guidelines and support that help to perform it in an easy way. We aim at a more generic modelling approach able to support any relevant point of view that makes sense for studying life trajectory. Second, the works $[17,8]$ do not handle clearly the ageographical (i.e. non geographic) dimension of some trajectories and make therefore no recommendation for mixing and integrating such trajectories with geographical ones in the same model. For these reasons, these models and ontological design patterns are not fully adapted to our problematic.

Furthermore the different concepts used to segment a trajectory in these approaches (stop, move, segment, fix, etc.) do not exactly carry the meaning we need to study: the multiple thematic points of view that belong to the same life trajectory. Our problematic requires representing the states of the individuals, according to the different points of view, but also providing a representation of the events between these states. This event representation allows characterizing the transitions between states. Further, we claim that some influences exist between states and events of a trajectory that led to the appearance of new events. Such influences are explanatory factors that can make a trajectory more understandable. This leads us to consider works dealing with event and explanatory factors representation.

\subsection{Modelling events and explanatory factors}

Beyond the description of the different states that compose a trajectory, there is a need for representing the events that have conducted to a change of state. In [10] the ODP Event Core ${ }^{4}$ is proposed as a core, minimalistic, and generic pattern for representing any event. Basically, an event here occurs at a Place and Time, has participants, and is associated with other "non-essential information" such as names, URIs, textual descriptions, etc. Illustrations that are given mainly relate to some cultural or sports events. The meaning given here to an event does not exactly correspond to what we need to represent in our approach. We use the term event in the sense of life events, such as a move, a promotion, a new job, a birth, etc. Thus, what we call Event is to be understood as a transition between two states.

The Event Ontology 5 (EO) is an upper level ontology used to describe events at a generic level similarly to [15]. An interesting point is that it includes a property factor that "relates an event to a passive factor" (in this ontology a factor is considered as passive since it is not an agent that takes part in the event). Conversely, the property called product relates an event with anything that could be considered as a consequence of this event. These properties, which are used to represent cause and effect, are very

4 http://ontologydesignpatterns.org/wiki/Community:EventCore

5 http://motools.sourceforge.net/event/event.html 
high-level properties: they have no range. It means they relate an event to any class as a factor. In the case study we have chosen, for a better understanding of the trajectory itself and an elicitation of the motivations that have led to a residential move, we propose to structure what can be explanatory factors of events. We claim that specific classes and properties could be defined in order to characterize different kinds of influence events may have in the life trajectory: the birth of a child (i.e. an event) has probably influenced the move (i.e. another event) of the family. This leads us to consider and differentiate whether the cause of an event is internal to the individual's trajectory, or external (it is exogenous) or related to her/his social network (i.e. depending on the trajectory of some other individuals she/he is strongly related with). Such a typology for representing cause of events intends to facilitate the analysis and the comparison of trajectories and their associated elements of explanation.

\section{Design patterns for explainable multiple trajectories}

\subsection{The Ontology Design Pattern Trajectories}

The Ontology Design Pattern Trajectories we propose is presented on figure 2. The main concepts that compose the trajectory are episode and event. An episode is a stable state seen from a thematic point of view during a time interval. A state is considered as stable when the values of all its descriptive attributes remain unchanged ${ }^{6}$. An event characterizes a transition between two stable states. Classes and relationships that compose the pattern are described below.

Trajectory. This class characterizes the trajectory of a given thematic point of view. It is linked to a foaf:person class through a property hastrajectory. The use of the well-known friend of a friend ${ }^{7}$ (foaf) ontology allows to link individuals with the proposed property. The use of this ontology is also a way to foster interoperability between our model and social networks. The trajectory is related to the episodes and events that compose it through the property hasEpisode and hasEvent.

\footnotetext{
${ }^{6}$ This modelling choice requires to choose carefully the information to represent (i.e. the relevant attributes), but also to determine the relevant degrees of precision. For instance, in our application case, regarding the rent of some accommodation, the precise amount might be less relevant than a range of values.

$7 \mathrm{http}: / / \mathrm{xmlns} . \mathrm{com} /$ foaf/spec/
} 


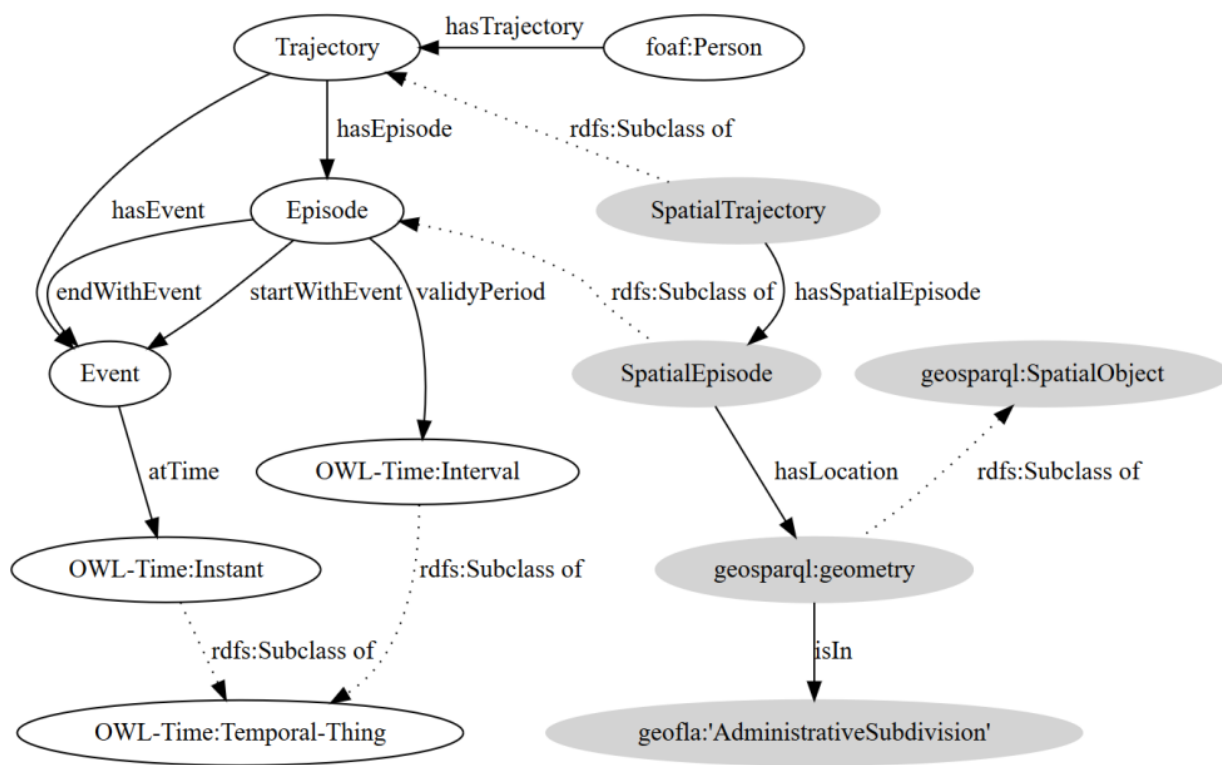

Figure 1: The Ontology Design Pattern for semantic trajectories

Episode. This class describes an episode of the trajectory. The property validityPeriod represents the episode duration that corresponds to a time interval between two instants $t_{s}$ and $t_{e}\left(a s t_{s}<t_{e}\right)$. Each of these two instants corresponds to an event in the trajectory: an episode starts and ends with an event (startWith and EndWi th properties). A non-strict order relation can be established between episodes using the algebra of Allen [Allen, 1983].

Event. This class characterizes an event of the trajectory. The property atTime associates an event with a chronon [1] relevant for the observed phenomenon (allowing to localize the event in time).

The temporal dimension of episodes and events is characterized using the OWLTime ontology ${ }^{8}$.

If the trajectory to model is a spatial trajectory, a more specifical class characterizing episodes has to be used, the class SpatialEpisode.

SpatialEpisode. This class is a specialization of the Episode class. Since it carries the spatial component of the trajectory, the spatial episode is connected to the geosparql : geometry class of the geosparql ontology ${ }^{9}$.

Finally, the location is related to the geofla ontology ${ }^{10}$ of the French National Geographic Institute that describes the French administrative subdivisions. The use of this ontology is optional and obviously only relevant for case studies located in France.

This design pattern has been implemented in RDF/Turtle.

\footnotetext{
8 https://www.w3.org/TR/owl-time/

${ }^{9} \mathrm{http}: / /$ schemas.opengis.net/geosparql/

${ }^{10} \mathrm{http} / / /$ data.ign.fr/def/geofla/20140822.en.htm
} 


\subsection{Explanatory factors of events}

The second design pattern that we propose allows modelling explanatory factors of events. In our approach, we consider that life events can be explained either:

- $\quad$ by other life events: for instance, because Thomas had a promotion (event), he moved for a bigger apartment (event), or

- $\quad$ by the characteristics of an episode: for instance, because Thomas' apartment was too small ( surface $=35 \mathrm{~m}^{2}$, episode's characteristics), he moved to a bigger one (event). Combinations of such explanations are obviously possible: for instance, because $i$ ) Thomas had a promotion (event), ii) he had a baby (event) and $\mathrm{iii}$ ) his apartment was too small (surface $=35 \mathrm{~m}^{2}$ and numberOfRoom $=1$, episode's characteristics), then he moved to a bigger apartment (event).

Our design pattern is presented in figure 3. The classes and properties of this design pattern are described below. In this representation the class Event corresponds to the event to be explained.

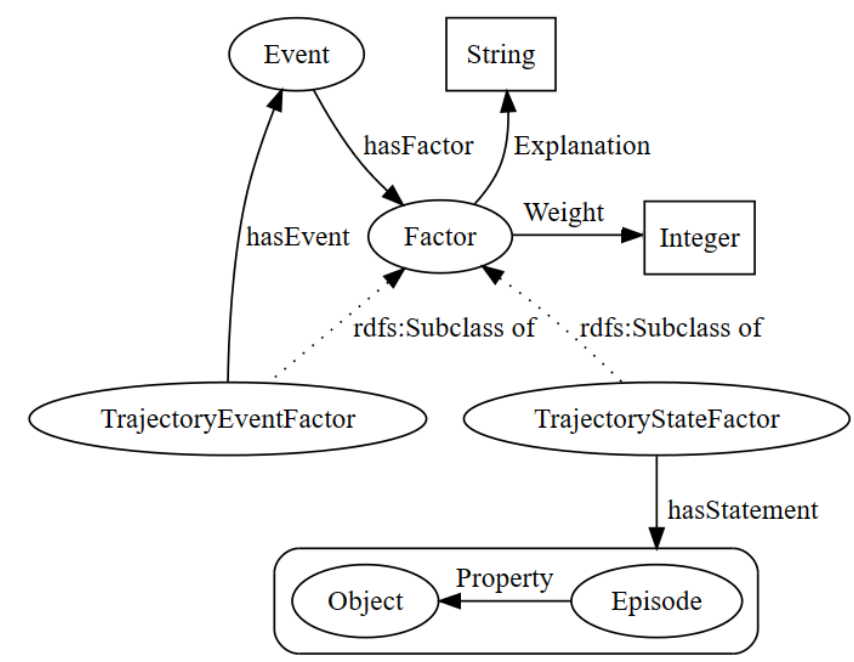

Figure 2: Design pattern for explanatory factors of trajectory events

Factor is a general class that characterizes an explanatory factor for an event. A factor is related to an event through a property hasFactor. The data property Explanation relates a Factor to a String that provides additional explanation about this particular factor, if needed. The data property Weight associates a Factor with an Integer that allows to express the weight of this particular factor on the explained event. When several explanatory factors exist for the same event, this is useful for a finer understanding of the influence of each.

The class Factor is subsumed by two classes: TrajectoryEventFactor and TrajectorystateFactor. 
TrajectoryEventFactor characterizes an explanatory factor that has an explaining event. This explaining event belongs to the trajectory of any individual. For instance, Thomas' move (the explained event) could be explained by his promotion at work (a factor - explaining event - from his professional trajectory) or by his wife's promotion at work (a factor - explaining event - from his wife's professional trajectory). A TrajectoryEventFactor is therefore related to an explaining event through a relationship hasEvent. This class has two sub-classes (not presented on the figure): InternalEventFactor and NetworkEventFactor for supporting the examples given above.

InternalEventFactor is related to an explaining event that belongs to the trajectory of the same individual. In the previous example, the promotion of Thomas is therefore characterized by the InternalEventFactor Class.

NetworkEventFactor is related to an explaining event that belongs to the trajectory of another individual. In the previous example, the promotion of Thomas'wife is characterized by the NetworkEventFactor Class. The two (possibly indirectly) related individuals form consequently (a part of) a network. When possible, such relationships are expressed using the foaf ontology.

TrajectorystateFactor characterizes an explanatory factor that has an explaining statement that describes a state in the trajectory of the individual. In our model, statements describing states are related to episodes. For instance, a residential move (the resulting event) is explained by some characteristics of the previous residential episode such as a too small number of rooms (the explaining statement). TrajectorystateFactor is therefore related to an explaining statement through a relationship has tatement. This property uses RDF reification, a mechanism that allows making a statement about another statement (i.e. triple subject-predicate-object altogether is assigned a URI and treated as a resource about which additional statements can be made).

\section{Exploitation of the design patterns}

In this section, we show how our design patterns can be exploited to create a life trajectory model that supports the representation of both multiple thematic component trajectories and explanatory factors.

\subsection{Modelling a first trajectory}

We present a simplified version of the algorithm that allows applying the proposed design pattern to create the first thematic trajectory of the model. The algorithm InitializeModelTrajectory creates a trajectory model (see Figure 3) for a given point of view and according to its nature (spatial or not). The Boolean isspatial is true if the trajectory is spatial, otherwise false. The String viewStamp is a relevant string for describing the point of view used to stamp the trajectory. For instance, we use the stamp "Residential" for the residential spatial trajectory. 
The HashTable EpisodeAttributes is a <<string,String>> HashTable that stores each attribute (AttName) and its associated type (AttType). These couples of information characterize the episode of the thematic trajectory. For example, for the residential point of view, relevant attributes could be HousingType (a string) and the NumberOfRooms (an integer).

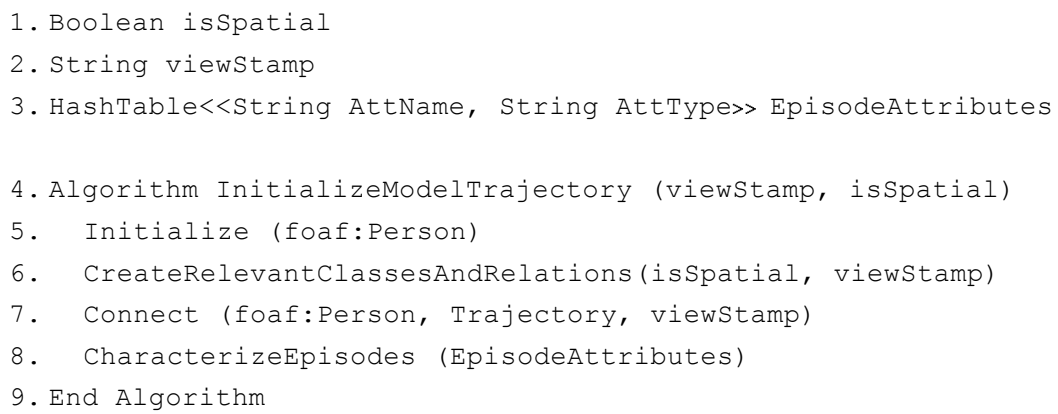

Algorithm 1 - InitializeModelTrajectory

The method Initialize (foaf:Person) (line 5) creates the foaf prefix in the ontology and initialize the foaf:Person class.

The method CreateRelevantClassesAndRelations(isspatial, viewStamp) (line 6) creates the classes and relationships using the relevant part of the pattern according to the value of the Boolean isspatial. If the Boolean valuates to false, the created classes are Trajectory, Episode and Event and the created relationships are hasTrajectory, hasEpisode, hasEvent, endwith, startwith. If the Boolean isspatial is true, the classes Spatialtrajectory and SpatialEpisode are created instead of the aspatial version. In both cases, each class and relationship of the pattern is renamed at its creation time by prefixing its name with the String viewstamp.

The method connect (foaf:Person, Trajectory, viewstamp) (line 7) creates a relationship between the foaf: Person class and the (just stamped) Trajectory class.

The method CharacterizeEpisodes (EpisodeAttributes) (line 8) creates, for each pair of the HashTable EpisodeAttributes, the relevant predicate whose domain is the stamped Episode (or SpatialEpisode) class. This method creates a data property. For instance, for the pair $\langle<$ NumberOfRoom, Integer $\rangle>$ the data property NumberOfRoom whose range is an Integer and whose domain is ResidentialspatialEpisode, is created (see Figure 3). 


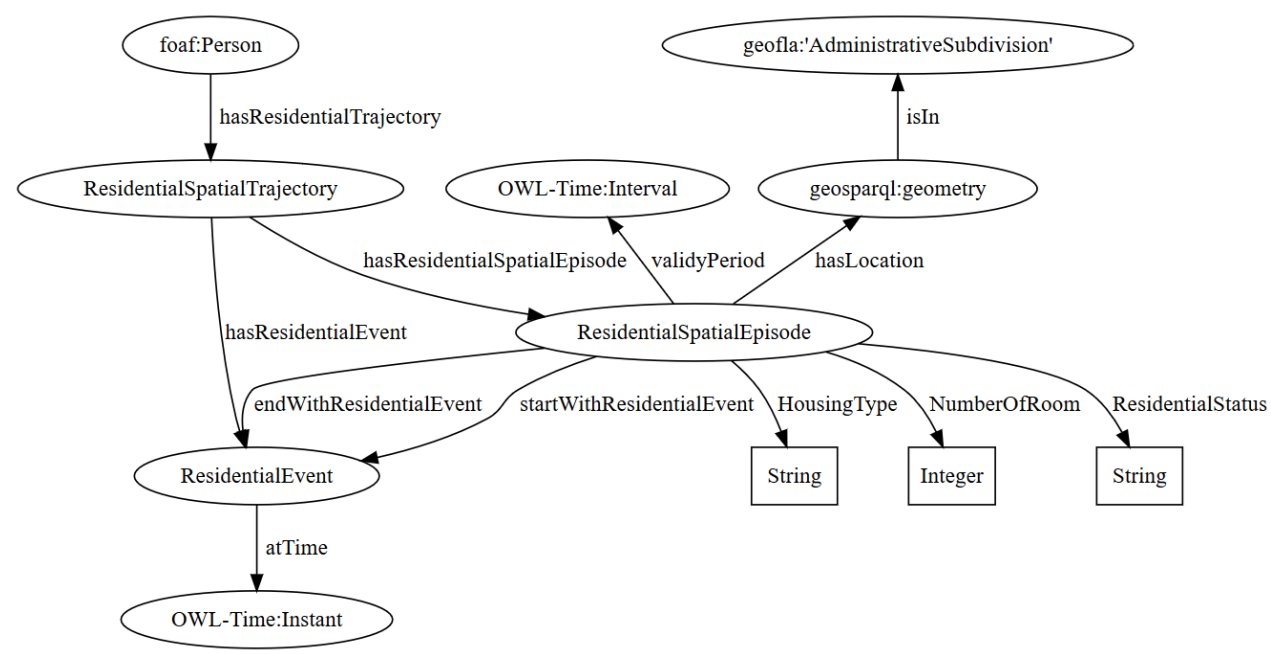

Figure 3: The residential trajectory

\subsection{Modelling multiple trajectories}

To build a more complex model that integrates the multiple points of view of a life trajectory, we propose a second algorithm. It works in the same way as for Algorithm 1 , except that it does not create a new instance of a foaf: Person but associates this new trajectory with the existing class. Thus, in addition to the variables used in the previous algorithm, this one is using a String PersonUri that is the URI of a previously created foaf: Person class.

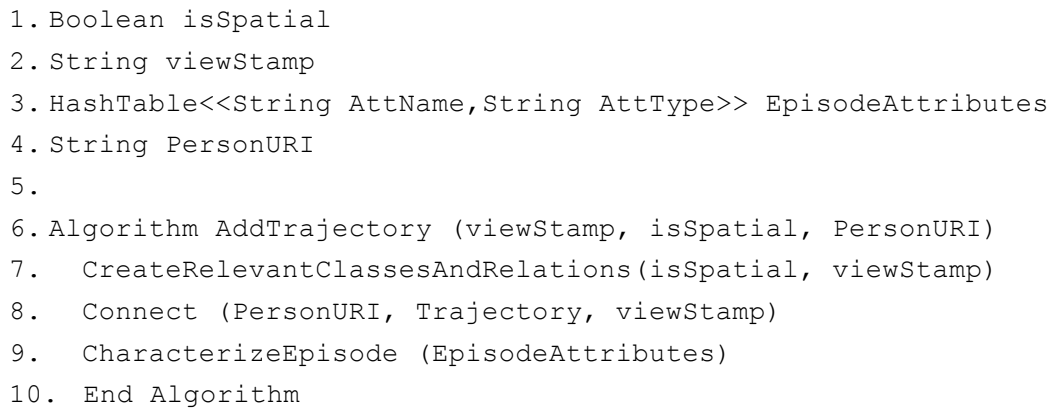

\section{Algorithm 2 - AddTrajectory}

Figure 4 illustrates how two thematic trajectories, respectively stamped with A and $\mathrm{B}$, are finally linked into the same model corresponding to the life trajectory of an individual. The class property hasFactor (see section 3.2) that relates an Event to a Factor can then be used for each stamped Event class. 


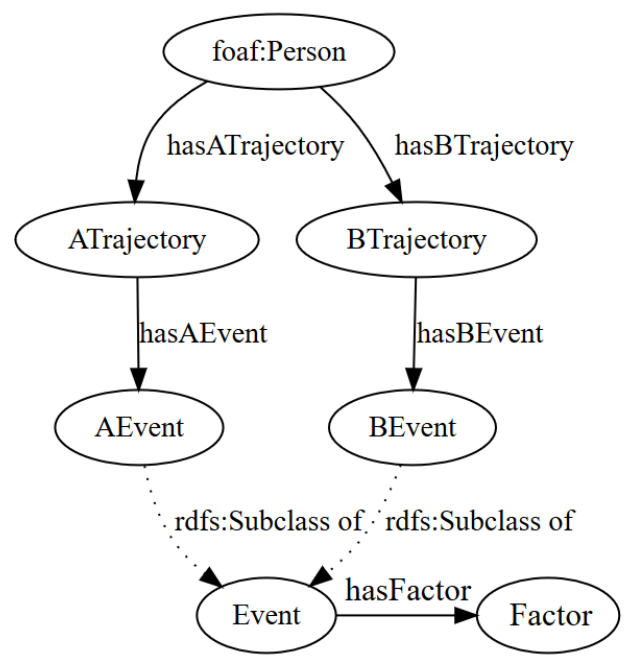

Figure 4: Modeling of two thematic point of view

In the next section, we show through a case study how such an ontology, produced using our patterns, can be exploited.

\section{Exploitation of the ontology}

\subsection{Application to residential trajectory data}

The life trajectory model obtained using the design pattern was tested to store life trajectory data related to the residential choices and their explanation. Data were collected during a survey among 30 individuals in the French city of Grenoble metropolitan area. A semi-directed interview was conducted with each individual. During the interview, people were asked to explain their own trajectory as precisely as possible in a chronological way. The interviewer followed survey guidelines that help to collect useful data. Collected data were then used to fulfil an ontology model built from our design patterns. Our objective was to test the validity of the produced ontology and its expressiveness. 


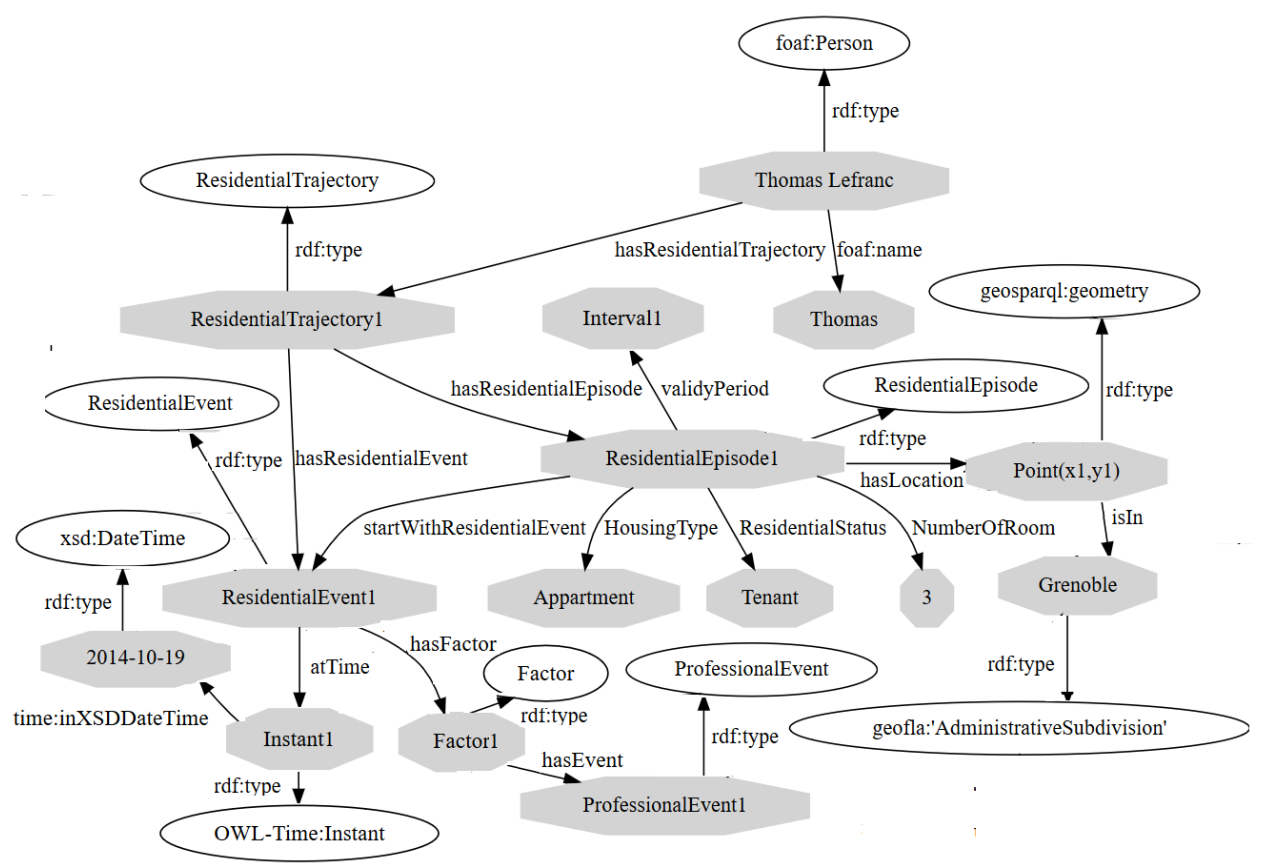

Figure 5: An example of annotated instance of the model

Figure 5 presents an instance of a residential trajectory compliant with the ontology shown in Figure 3. This is an excerpt of Thomas' residential trajectory (ResidentialTrajectory 1) that includes a residential episode (ResidentialEpisode1) that started with Thomas' move (ResidentialEvent1) on 2014-10-19. During this residential episode, Thomas was a tenant of a three-room apartment. We know the location of the housing (hasLocation) that is in the Grenoble administrative division, in France. The model also tells us that the ResidentialEpisode1 started with (startwith) the ResidentialEvent1 whose one of the factors is a professional event (ProfessionalEvent1, a promotion, not shown here).

\subsection{Querying residential trajectory model}

Once filled, the ontology can be exploited to query the multiple trajectories.

The multiple semantic points of view that compose the whole trajectory can be queried together. With respect to our case study, we give some queries example. The life trajectory model has been implemented in the parliament triple store ${ }^{11}$ and the queries

${ }^{11} \mathrm{http} / / /$ parliament.semwebcentral.org/ 
have been tested using the provided SPARQL endpoint. The prefix LTO (Life Trajectory Ontology) was used for this example. These queries allow to access the relevant resource(s) which are identified with an URI.

Q1: What are the explanatory factors of the residential event ResidentialEvent1 and what is the explanation associated with these factors?

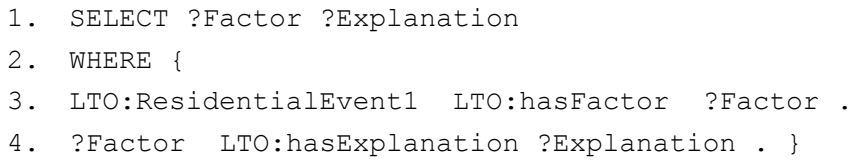

This query returns the URI that corresponds to Factor 1 and the associate explanation (see Figure 1). From Factor1, one could navigate to the associated event (here ProfessionalEvent1).

Q2: Which residential events were influenced by a professional event?

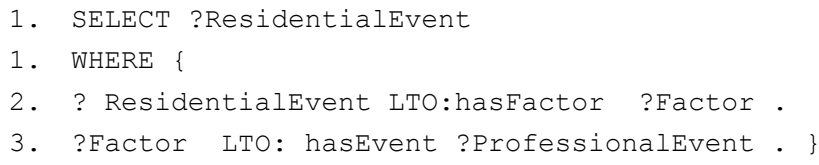

Q3: Which are the residential trajectories that pass through Grenoble?

Such a query illustrates how geographical information is used. The Grenoble entity is an instance of the class commune (and an administrative subdivision) in the French geofla ontology. The label of the Grenoble entity is "GRENOBLE"@fr. The geofla ontology and data on the administrative subdivision are available as linked data on the open data portal of the French National Geographic Institute ${ }^{12}$ (IGN). The following query uses a SPARQL service to request the SPARQL endpoint ${ }^{13}$ available on this portal.

1. SELECT ?ResidentialTrajectory

2. WHERE \{

3. ?ResidentialTrajectory LTO:hasResidentialEpisode ?ResidentialEpisode.

4. ResidentialEpisode LTO:hasLocation ?Location .

5. ?Location LTO:isIn ?commune.

6. SERVICE <http://data.ign.fr/id/sparql> \{ ?commune rdfs:label "GRENOBLE"@fr . \} \}

Q4: What are the explanatory factors of the residential move to Grenoble after 2014? This spatio-temporal query involves the OWL-Time ontology and a SPARQL filter.

\footnotetext{
${ }^{12} \mathrm{http}: / /$ data.ign.fr/

${ }^{13} \mathrm{http} / / /$ data.ign.fr/id/sparql/
} 


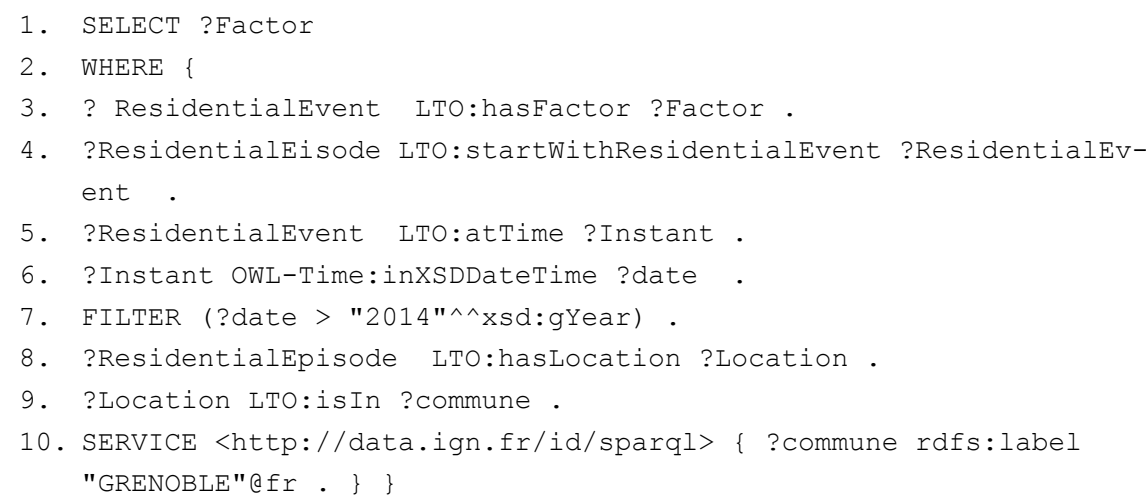

\section{Conclusion and future work}

In this paper, we have proposed two ontological design patterns that allow modelling multiple spatial or aspatial trajectories and that include explanatory factors for a better understanding of trajectory events. The proposed ontological design patterns are related to several upper level ontologies in order to allow interoperability of the produced models with the web of data. We have also provided algorithms that exploit our patterns to build ontologies. As a case study, we have modelled the multiple trajectories that compose a life trajectory having in mind to study residential choices. Finally, we have shown how the trajectory model we get can be exploited using temporal, spatial and thematic dimensions using the SPARQL language.

One possible perspective of our work is to rebuild a model from the analysis of existing trajectories. The STEP ontology [12] propose to structure trajectories into spatiotemporal episodes gathering together raw data that share some characteristics. We may not manipulate the same kind of data (they use raw data concerning joggers runnings) but the adaptation of their principles to our context could lead to make some coarsergrain episodes emerge from a life trajectory. To achieve this, we will need to design algorithms exploiting data at multiple granularity levels using the connected upper level ontologies (OWL-Time and geosparl).

\section{$7 \quad$ Acknowledgment}

We would like to thank the Auvergne-Rhône-Alpes Region Council for his support (D. Noël PhD Grant). 


\section{$8 \quad$ References}

1. Allen, J. F. (1983). Maintaining knowledge about temporal intervals. Communications of the ACM, 26(11), 832-843.[3] Alvares, L. O., Bogorny, V., Kuijpers, B., de Macelo, J. A. F., Moelans, B., \& Palma, A. T. (2007). Towards semantic trajectory knowledge discovery. Data Mining and Knowledge Discovery.

2. Authier, J. Y., Bidet, J., Collet, A., Gilbert, P., \& Steinmetz, H. (2012). État des lieux sur les trajectoires résidentielles.

3. Battle, R., \& Kolas, D. (2011). Geosparql: enabling a geospatial semantic web. Semantic Web Journal, 3(4), 355-370.

4. Becker, H (1963). Outsiders: studies in the sociology of deviance.

5. Bonvalet, C., Laflamme, V., \& Arbonville, D. (2009). Family and housing: recent trends in France and Southern Europe. Bardwell Press.

6. Grafmeyer, Y., \& Authier, J. Y. (2011). Sociologie urbaine. Armand colin. IN FRENCH

7. Hägerstraand, T. (1970). What about people in regional science?. Papers in regional science, 24(1), 7-24.

8. Hu, Y., Janowicz, K., Carral, D., Scheider, S., Kuhn, W., Berg-Cross, G., ... \& Kolas, D. (2013, September). A geo-ontology design pattern for semantic trajectories. In International Conference on Spatial Information Theory (pp. 438-456). Springer International Publishing.

9. Krisnadhi, A.A.: Ontology Pattern-Based Data Integration. Ph.D. thesis, Department of Computer Science and Engineering, Wright State University (2015)

10. Krisnadhi, A.A., and Hitzler, P., A Core Pattern for Events Workshop on Ontology and Semantic Web Patterns (7th edition) - WOP2016, Kobe, Japan, $18^{\text {th }}$ October, 2016.

11. Krisnadhi, A.A., Hitzler, P., Janowicz, K.: A Spatiotemporal Extent Pattern based on Semantic Trajectories, Workshop on Ontology and Semantic Web Patterns (7th edition) WOP2016, Kobe, Japan, $18^{\text {th }}$ October, 2016.

12. Nogueira T.P., Martin H., "Querying Semantic Trajectory Episodes" in 4th ACM SIGSPATIAL International Workshop on Mobile Geographic Information Systems (MobiGIS'15), 2015.

13. OGC. (2012). OGC GeoSPARQL - A Geographic Query Langage for RDF Data.

14. Raimond, Y. et S. Abdallah.: The Timeline Ontology. http://motools.sourceforge.net/timeline/timeline.html (2007)

15. Raimond, Y., Abdallah, S.: The Event Ontology. Available online from http://motools.sourceforge.net/event/event.html (2007)

16. Raubal, M. (2008, September). Representing concepts in time. In International Conference on Spatial Cognition (pp. 328-343). Springer Berlin Heidelberg.

17. Spaccapietra, S., Parent, C., Damiani, M. L., de Macedo, J. A., Porto, F., \& Vangenot, C. (2008). A conceptual view on trajectories. Data \& knowledge engineering, 65(1), 126-146.

18. Thériault, M., Claramunt, C., Séguin, A. M., \& Villeneuve, P. (2002). Temporal GIS and statistical modelling of personal lifelines. In Advances in Spatial Data Handling (pp. 433449). Springer Berlin Heidelberg.

19. Thériault, M., Séguin, A. M., Aubé, Y., \& Villeneuve, P. Y. (1999). A spatio-temporal data model for analysing personal biographies. In Database and Expert Systems Applications, 1999. Proceedings. Tenth International Workshop on (pp. 410-418). IEEE.

20. Zheni, D., Frihida, A., Ghezala, H. B., \& Claramunt, C. (2009). A semantic approach for the modeling of trajectories in space and time. In Advances in Conceptual Modeling-Challenging Viewpoints (pp. 347-356). Springer Berlin Heidelberg. 